\title{
Effects of isomaltulose on insulin resistance and metabolites in patients with non-alcoholic fatty liver disease: A metabolomic analysis
}

\author{
TAKUMI KAWAGUCHI, DAN NAKANO, TETSUHARU ORIISHI and TAKUJI TORIMURA \\ Division of Gastroenterology, Department of Medicine, \\ Kurume University School of Medicine, Kurume, Fukuoka 830-0011, Japan
}

Received March 19, 2018; Accepted June 20, 2018

DOI: $10.3892 / \mathrm{mmr} .2018 .9223$

\begin{abstract}
Insulin resistance is associated with a poor prognosis in non-alcoholic fatty liver disease (NAFLD) patients. Isomaltulose, a naturally-occurring disaccharide, is reported to improve glucose and lipid metabolisms in obese patients. The present study aimed to investigate the effects of isomaltulose on insulin resistance and various metabolites in NAFLD patients. Five male patients with NAFLD consumed $20 \mathrm{~g}$ isomaltulose or sucrose (control). Changes in insulin resistance and metabolites were evaluated by alterations of serum C-peptide immunoreactivity (CPR) and metabolomic analysis from baseline to $15 \mathrm{~min}$ after the administration, respectively. There was no significant difference in changes of blood glucose level; however, the CPR level was significantly decreased in the Isomaltulose group compared to the control group $(0.94 \pm 0.89$ vs. $-0.12 \pm 0.31, P=0.0216)$. In a metabolomic analysis, a significant alteration was seen in 52 metabolites between the control and Isomaltulose groups. In particular, the taurodeoxycholic acid level significantly increased approximately 12.5 -fold, and the arachidonic acid level significantly
\end{abstract}

Correspondence to: Dr Takumi Kawaguchi, Division of Gastroenterology, Department of Medicine, Kurume University School of Medicine, 67 Asahi-machi, Kurume, Fukuoka 830-0011, Japan

E-mail: takumi@med.kurume-u.ac.jp

Abbreviations: NAFLD, non-alcoholic fatty liver disease; GLP-1, glucagon-like-peptide 1; GIP, glucose-dependent insulinotropic polypeptide; 6-0- $\alpha$-D-glucopyranosyl-D-fructose, Isomaltulose; AST, aspartate aminotransferase; ALT, alanine aminotransferase; LDH, lactate dehydrogenase; GGT, $\gamma$-glutamyl transpeptidase; LDL, low-density lipoprotein; BUN, blood urea nitrogen; CPR, C-peptide immunoreactivity; LC-MS, liquid chromatography mass spectrometry; CE-MS, capillary electrophoresis coupled to mass spectrometry; TOFMS, time-of-flight mass spectrometry; IRI, immunoreactive insulin

Key words: isomaltulose, non-alcoholic steatohepatitis, metabolomics, insulin resistance, bile acids, fatty acids decreased approximately 0.01 -fold. Together, it present study demonstrated that isomaltulose improved insulin resistance in NAFLD patients. It was also revealed that isomaltulose affects taurodeoxycholic acid and arachidonic acid. Thus, isomaltulose may have a beneficial effect on insulin resistance through alterations of bile acid and fatty acid metabolisms in NAFLD patients.

\section{Introduction}

Non-alcoholic fatty liver disease (NAFLD) is a pandemic disease, and one-third of the population worldwide is affected $(1,2)$. Since the liver is one of the target organs of insulin, a high prevalence of insulin resistance is seen in patients with NAFLD $(3,4)$. An increase in insulin resistance is associated with the development of not only type 2 diabetes mellitus but also life-threatening diseases such as liver cirrhosis, hepatocellular carcinoma, and cardiovascular diseases in patients with NAFLD (5). Thus, improved insulin resistance is an important therapeutic target in patients with NAFLD. Anti-diabetic agents including pioglitazone (6), glucagon-like peptide-1 (GLP-1) analogue (7), and sodium-glucose cotransporter 2 inhibitor (8) lead to metabolic and histologic improvement in patients with NAFLD complicated by diabetes mellitus. However, no effective medication has been approved yet for patients with NAFLD.

Isomaltulose (6-0- $\alpha$-D-glucopyranosyl-D-fructose) is a naturally-occurring disaccharide found in honey (9) and is composed of glucose and fructose, similar to sucrose (10). Both isomaltulose and sucrose are digested into glucose and fructose by $\alpha$-glucosidase in the small intestine and contribute the same caloric value of $4 \mathrm{kcal} / \mathrm{g}(11,12)$. However, the digestive rate of isomaltulose is significantly slower than that of sucrose because the structural feature of isomaltulose is that glucose and fructose are linked by an alpha-1,6-glycosidic bond $(12,13)$. In addition, isomaltulose affects the secretion of gut hormones such as glucose-dependent insulinotropic polypeptide (GIP) and GLP-1 (14,15), leading to an improvement of insulin resistance. Thus, isomaltulose is a low glycemic index sweetener as well as a functional disaccharide and is currently used in various medical food and drink applications instead of sucrose (16). 
In a rat model of metabolic syndrome, isomaltulose is reported to reduce visceral fat mass and improves glucose intolerance, resulting in inhibition of increase in blood pressure and progression of diabetic nephropathy (17). Furthermore, in healthy subjects, isomaltulose ingestion inhibits an increase in insulin resistance and blood pressure $(11,18)$. The beneficial effects of isomaltulose on visceral fat, insulin resistance, and blood pressure in patients who were obese have been reported in double-blind, placebo-controlled interventional studies $(19,20)$. However, the effect of isomaltulose on insulin resistance has never been investigated in patients with NAFLD.

An increase in insulin resistance can be caused by various factors including amino acid, fatty acid, and bile acid metabolism $(3,21,22)$. Metabolomic analysis is a systematic examination of metabolites in a given biological sample and can reveal novel pathways (23). Metabolomic analysis has recently been applied to the identification of the pharmacological mechanisms of berberine, an isoquinoline alkaloid, for nonalcoholic steatohepatitis (NASH) treatment and revealed metabolic disruption involving phospholipids and unsaturated fatty acids in a rat model of NASH (24). However, metabolomic analysis has not been used to identify the pharmacological mechanisms of isomaltulose in patients with NAFLD.

The aim of this study was to investigate effects of isomaltulose on insulin resistance in patients with NAFLD. In addition, using metabolomic analysis, we investigated the effect of isomaltulose on various metabolisms in patients with NAFLD.

\section{Subjects and methods}

Study design and ethics. This was a randomized, single-blinded controlled interventional study, and our study protocol conformed to the ethical guidelines of the 1975 Declaration of Helsinki, as reflected by the prior approval of the institutional review board of The Ethic Committee of Kurume University. All experiments were performed in accordance with relevant guidelines and regulations. All subjects provided written informed consent to participate in this study.

Subjects. A total of 5 male patients diagnosed with NAFLD were enrolled in this study. The following patient inclusion criteria were used: Patients with i) NAFLD, ii) age $>20$ years, and iii) written informed consent. The exclusion criteria were as follows: Patients with i) hemoglobin Alc $>8.0 \%$,ii) insulin or $\alpha$-glucosidase inhibitor treatment, iii) liver cirrhosis, iv) renal failure, v) a history of cardiovascular disease, or vi) participation in any other clinical trial.

The subjects were randomly assigned into the sucrose (Control) or isomaltulose group. After a 14-day wash-out term, each subject was assigned to the other group.

Diagnosis of NAFLD. NAFLD was diagnosed according to the Clinical Practice Guidelines for NAFLD/NASH as follows (25): i) hepatic steatosis evaluated by liver biopsy, ultrasonography, computed tomography, or magnetic resonance imaging; ii) ethanol intake $<30 \mathrm{~g} /$ day; and iii) exclusion of other liver diseases, including chronic hepatitis B, chronic hepatitis $\mathrm{C}$, autoimmune hepatitis, drug-induced liver disease, primary biliary cholangitis, primary sclerosing cholangitis, biliary obstruction, Wilson's disease, and hemochromatosis.
Isomaltulose or sucrose administration and blood collection. After a 12-hour overnight fast, the subjects consumed $20 \mathrm{~g}$ isomaltulose in $200 \mathrm{ml}$ of water or $20 \mathrm{~g}$ sucrose (Control) in $200 \mathrm{ml}$ of water in $1 \mathrm{~min}$ at 8:30 a.m. During the procedure, the subjects were instructed to rest on a bed and fast. Venous blood samples were collected before and 15 min after administration. For plasma GIP and GLP-1 measurements, blood samples were collected into a $\mathrm{BD}^{\mathrm{TM}} \mathrm{P} 100$ Blood Collection System containing protein stabilizers (BD Biosciences, Franklin Lakes, NJ, USA). Blood samples were centrifuged at $3,000 \mathrm{x} \mathrm{g}$ for $15 \mathrm{~min}$ at $4^{\circ} \mathrm{C}$ and stored at $-20^{\circ} \mathrm{C}$ until analysis.

Laboratory determinations. Serum levels of aspartate aminotransferase (AST), alanine aminotransferase (ALT), lactate dehydrogenase (LDH), $\gamma$-glutamyl transpeptidase (GGT), total bilirubin, total cholesterol, low-density lipoprotein (LDL)-cholesterol, blood urea nitrogen (BUN), creatinine, nonesterified fatty acid, C-peptide immunoreactivity (CPR), and blood glucose level were measured using standard clinical methods (SRL Inc., Tokyo, Japan) as previously described $(4,26,27)$.

Measurement of serum GIP and GLP-1 levels. As previously described (28), plasma GIP and GLP-1 levels were measured by an enzyme-linked immunosorbent assay using a Human GIP ELISA kit (YK253; Yanaihara Institute Inc., Fujimiya, Japan) and a Human GLP1 (7-36) ELISA kit (ab184857; Abcam, Cambridge, UK), respectively, according to each manufacturer's instructions.

Metabolomic analysis. Metabolomic analysis was performed twice using serum samples taken before $(0 \mathrm{~min})$ and $15 \mathrm{~min}$ after isomaltulose or sucrose treatment.

Preparation. Serum samples taken before and 15 min after isomaltulose or sucrose treatment from a representative subject were applied to a metabolomic analysis. Metabolome measurements were performed at a service facility of LSI Medience Corporation (Tokyo, Japan). Briefly, serum (200 $\mu \mathrm{l})$ was added to methanol $(800 \mu \mathrm{l})$ and then mixed for $15 \mathrm{~min}$ with a shaker at room temperature. After it was centrifuged by $10,000 \mathrm{x} \mathrm{g}$ for $10 \mathrm{~min}$, the supernatant was dried up with nitrogen gas, and the residue was dissolved with $10 \%$ acetonitrile aqueous solution $(200 \mu \mathrm{l})$. After adding internal standards, they were analyzed with both liquid chromatography mass spectrometry (LC-MS) and capillary electrophoresis coupled to mass spectrometry (CE-MS). Tuning and calibration were performed with a standard solution provided by Agilent Technology, and the resolution errors were controlled within $3 \mathrm{ppm}$. The order of measurement was randomized to minimize the specific error in each group. Quality control samples were prepared by pooling samples and were analyzed every 5 samples to verify the measurement accuracy.

LC-time-of-flight mass spectrometry (TOFMS) analysis. LC-MS datasets were acquired on a liquid chromatography system (Agilent HP1200; Agilent Technologies, Inc., Santa Clara, CA, USA) equipped with a C18 column $(2 \mu \mathrm{m}$, 50x2.0 mm ID, CAPCELL PAK C18 IF; Shiseido, Tokyo, Japan) and coupled with an electrospray ionization quadrupole 
TOFMS (Agilent 6520; Agilent Technologies, Inc.). Solvent A was composed of a $5 \mathrm{mM}$ ammonium acetate aqueous solution, while solvent B was acetonitrile. Metabolites were eluted at a flow rate of $0.2 \mathrm{ml} / \mathrm{min}$ at $40^{\circ} \mathrm{C}$ with a linear gradient of $10-90 \%$ solvent B over $10 \mathrm{~min}$, followed by a further $5 \mathrm{~min}$ hold at $100 \%$ solvent $\mathrm{B}$. The mass spectrometer was operated in positive and negative scan mode $(\mathrm{m} / \mathrm{z} 60$ to 1,200$)$ with a capillary voltage of $3,500 \mathrm{~V}$. The nebulizing gas pressure was $40 \mathrm{psi}$, and the dry gas flow was $81 / \mathrm{min}$ at $350^{\circ} \mathrm{C}$.

CE-TOFMS analysis. Ionic metabolites were measured in the positive mode of CE-TOFMS (Agilent CE-TOFMS 6520; Agilent Technologies, Inc.,). Metabolites were separated in a fused-silica capillary $(50 \mu \mathrm{m}$ i.d.x $100 \mathrm{~cm}$ total length; GL Science, Tokyo, Japan) filled with $1 \mathrm{~mol} / 1$ formic acid aqueous solution (cation mode), or $20 \mathrm{mM}$ ammonium formate and $20 \mathrm{mM}$ ammonium acetate aqueous solution $(\mathrm{pH} 10$, anion mode) as an electrolyte. The sample solution was injected at $5 \mathrm{kPa}$ for $15 \mathrm{sec}$ (approximately $15 \mathrm{nl}$ ), and a voltage of $30 \mathrm{kV}$ was applied. The temperatures of capillary and sample trays were maintained at room temperature and $5^{\circ} \mathrm{C}$, respectively. The sheath liquid was methanol/water $(50 \% \mathrm{v} / \mathrm{v})$ containing $5 \mathrm{mM}$ ammonium acetate. CE-TOFMS was operated in both positive and negative scan mode (m/z 60 to 1,200). The capillary voltage was set at $3,500 \mathrm{~V}$, and the nitrogen gas (heater temperature $250^{\circ} \mathrm{C}$ ) flow rate was set at $101 / \mathrm{min}$.

Data analysis. A data file of mass spectrometry was converted to csv format with an Agilent csv converter. All peak positions (retention time and $\mathrm{m} / \mathrm{z}$ ) and areas were calculated by Markeranalysis (LSI Medience, Tokyo, Japan) (29). All peak areas were aligned into one data sheet, and the errors of peak intensities were corrected by internal standards. Noise peaks were deleted compared with the peaks detected in blank samples. Metabolites were identified by comparing the retention time and $\mathrm{m} / \mathrm{z}$ with standard data set established by LSI Medience Corporation.

Effects of isomaltulose on insulin resistance, GIP, GLP-1 secretion, and metabolites. Insulin resistance was evaluated by an increment of serum CPR as previously described (30). $\triangle \mathrm{CPR}$ was the change of serum CPR level from baseline to $15 \mathrm{~min}$ after sucrose or isomaltulose administration. Similarly, changes in GIP and GLP-1 secretions were evaluated by $\triangle$ GIP and $\triangle$ GLP-1, which were changes of plasma GIP and GLP-1 levels from baseline to $15 \mathrm{~min}$ after sucrose or isomaltulose administration, respectively. The effects of isomaltulose on metabolites were also evaluated by change of each metabolite level from baseline ( $0 \mathrm{~min}$ ) to $15 \mathrm{~min}$ after sucrose or isomaltulose administration ( $\Delta$ each metabolite). Variable that increased or decreased after the administration was shown as positive or negative expression level ( $\Delta$ variable or $-\Delta$ variable) (Fig. 1$)$.

Statistical analysis. Data are expressed as numbers or means \pm standard deviations. Differences between the two groups were analyzed using the Wilcoxon signed-rank test. In metabolome analysis, mea $\mathrm{n}$ fold-change and t-test for all detected peaks were performed between the two groups. $\mathrm{P}<0.05$ was considered to indicate a statistically significant difference.

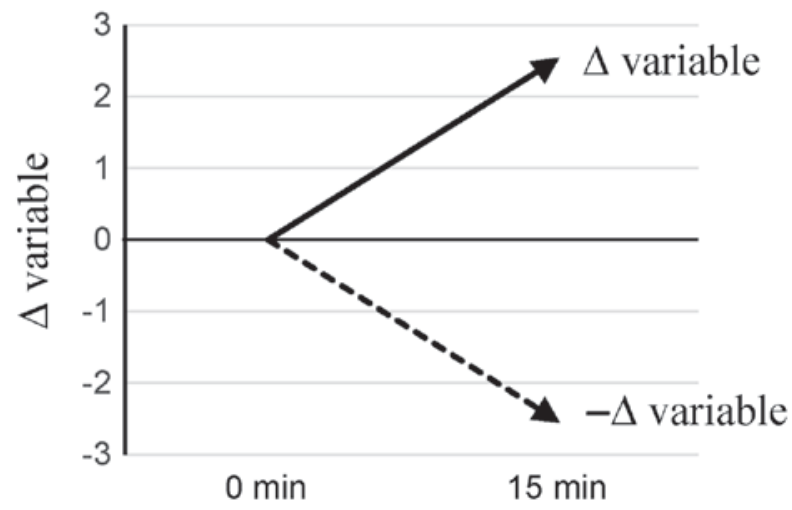

Figure 1. Changes in variable after administration of sucrose or isomaltulose. Effects of sucrose or isomaltulose on variables were evaluated by change of each variable level from baseline $(0 \mathrm{~min})$ to $15 \mathrm{~min}$ after administration. Variable that increased or decreased after the administration was shown as positive or negative expression level ( $\Delta$ variable or $-\Delta$ variable).

\section{Results}

Baseline patient characteristics. The baseline patient characteristics are summarized in Table I. No significant difference was seen in the serum levels of AST, ALT, GGT, or total bilirubin between the sucrose (Control) and Isomaltulose groups. There was no significant difference in fasting blood glucose or serum CPR levels between the 2 groups (Table I).

Effect of isomaltulose on changes in blood glucose and serum CPR levels. The effect of isomaltulose on blood glucose and serum CPR levels was evaluated by changes in these variables from baseline to $15 \mathrm{~min}$ after sucrose or isomaltulose administration. Although there was no significant difference in changes of blood glucose levels, the serum CPR level was significantly decreased in the Isomaltulose group compared to that in the Control group (Fig. 2).

Effect of isomaltulose on changes in plasma GIP and GLP-1 levels. The effect of isomaltulose on plasma GIP and GLP-1 levels was evaluated by changes in these variables from baseline to $15 \mathrm{~min}$ after sucrose or isomaltulose administration. There was no significant difference in changes of plasma GIP and GLP-1 levels between the Control and Isomaltulose groups (Table II).

Effect of isomaltulose on serum metabolite levels. With metabolomic analysis, the effects of isomaltulose on 201 metabolite levels were evaluated by changes in these variables from baseline to $15 \mathrm{~min}$ after sucrose or isomaltulose administration. A significant alteration was seen in 52 metabolites between the Control and Isomaltulose groups (Table III). Many of these 52 altered metabolites were categorized as bile acid (6 metabolites), fatty acid (6 metabolites), or glycine/serine metabolism (4 metabolites) (Fig. 3).

A representative change of metabolite in each category was an increased taurodeoxycholic acid level in the bile acid category, a decreased arachidonic acid level in the fatty acid category, and an increased betaine level in the glycine/serine category after isomaltulose treatment (Fig. 4A-C). In addition, 
Table I. Patients' characteristics at baseline.

\begin{tabular}{|c|c|c|c|}
\hline Characteristic & Control & Isomaltulose & P-value \\
\hline Age (yeare) & $48.6 \pm 11.8$ & $48.6 \pm 11.8$ & 1.0000 \\
\hline Body mass index & $35.1 \pm 7.0$ & $35.1 \pm 7.0$ & 1.0000 \\
\hline Red blood cells $\left(\times 10^{4} / \mu 1\right)$ & $509.6 \pm 70.6$ & $514.4 \pm 59.7$ & 0.9105 \\
\hline Hemoglobin (g/dl) & $15.5 \pm 1.8$ & $15.6 \pm 1.3$ & 0.9540 \\
\hline White blood cells $(/ \mu 1)$ & $8,500 \pm 2,515$ & $7,600 \pm 2,531$ & 0.5882 \\
\hline Platelet count $\left(\times 10^{4} / \mu 1\right)$ & $29.5 \pm 4.1$ & $28.7 \pm 4.2$ & 0.7634 \\
\hline Aspartate aminotransferase (IU/l) & $31.2 \pm 13.2$ & $30.0 \pm 14.7$ & 0.8953 \\
\hline Alanine aminotransferase (IU/l) & $45.6 \pm 36.9$ & $40.2 \pm 28.5$ & 0.8024 \\
\hline Lactate dehydrogenase (IU/l) & $227.2 \pm 109.8$ & $237.2 \pm 108.4$ & 0.8884 \\
\hline$\gamma$-glutamyl transpeptidase (IU/l) & $55.6 \pm 39.9$ & $54.4 \pm 36.0$ & 0.9614 \\
\hline Total bilirubin (mg/dl) & $0.52 \pm 0.18$ & $0.46 \pm 0.18$ & 0.6130 \\
\hline Total cholesterol (mg/dl) & $207.0 \pm 26.4$ & $197.2 \pm 21.1$ & 0.5350 \\
\hline Low-density lipoprotein cholesterol (mg/dl) & $138.6 \pm 17.6$ & $125.0 \pm 23.8$ & 0.3362 \\
\hline Triglycerides (mg/dl) & $144.2 \pm 51.4$ & $154.2 \pm 56.5$ & 0.7772 \\
\hline Blood urea nitrogen (mg/dl) & $14.7 \pm 2.9$ & $14.7 \pm 4.2$ & 0.9864 \\
\hline Creatinine (mg/dl) & $0.85 \pm 0.16$ & $0.80 \pm 0.20$ & 0.6998 \\
\hline Fasting blood glucose (mg/dl) & $132.0 \pm 38.7$ & $145.0 \pm 50.0$ & 0.6586 \\
\hline C-peptide immunoreactivity (ng/dl) & $2.38 \pm 0.99$ & $2.61 \pm 1.71$ & 0.8025 \\
\hline Nonesterified fatty acid $(\mu \mathrm{Eq} / \mathrm{l})$ & $675.0 \pm 316.8$ & $574.4 \pm 221.0$ & 0.5782 \\
\hline
\end{tabular}

Data are expressed as mean $\pm \mathrm{SD}$. Differences between the two groups were analyzed using Wilcoxon signed-rank test. P-values of $<0.05$ are considered significant.

Table II. Effects of isomaltulose on changes in plasma GIP and GLP-1 levels.

\begin{tabular}{lccr}
\hline Level & Control & Isomaltulose & P-value \\
\hline$\Delta$ GIP & $23.1 \pm 25.6$ & $14.0 \pm 9.5$ & 0.8345 \\
$\Delta$ GLP-1 & $-51.5 \pm 98.0$ & $166.7 \pm 66.6$ & 0.1437
\end{tabular}

Data are expressed as mean $\pm \mathrm{SD}$. $\Delta \mathrm{GIP}$ and $\triangle \mathrm{GLP}-1$ are changes of plasma GIP and GLP-1 levels from baseline to 15 min after sucrose (Control) or isomaltulose administration, respectively. Differences between the 2 groups were analyzed using the Wilcoxon signed-rank test. P-values of $<0.05$ are considered significant. GIP, glucose-dependent insulinotropic polypeptide; GLP-1, glucagon-like peptide-1.

a significant decrease was seen in the serum L-arginine level in the isomaltulose group compared to that in the Control group (Fig. 4D), while the serum L-ornithine level was significantly increased in the isomaltulose group compared to that in the Control group (Fig. 4E).

\section{Discussion}

In this study, we demonstrated that isomaltulose improved insulin resistance in patients with NAFLD. Although isomaltulose did not have a significant effect on the changes in serum GIP and GLP-1 levels, we showed that isomaltulose significantly affects various metabolites, in
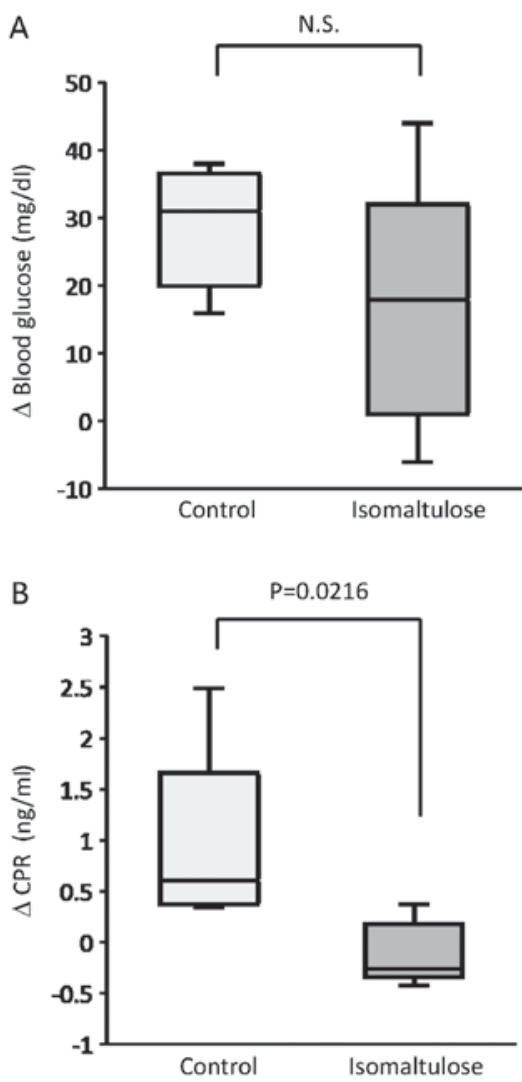

Figure 2. Effect of isomaltulose on changes in blood glucose and serum CPR levels. The effect of isomaltulose on blood glucose (A) and serum CPR levels (B) was evaluated by changes in these variables from baseline to $15 \mathrm{~min}$ after sucrose (Control) or isomaltulose administration. P-values of $<0.05$ were considered significant. N.S., not significant; CPR, C-peptide immunoreactivity. 







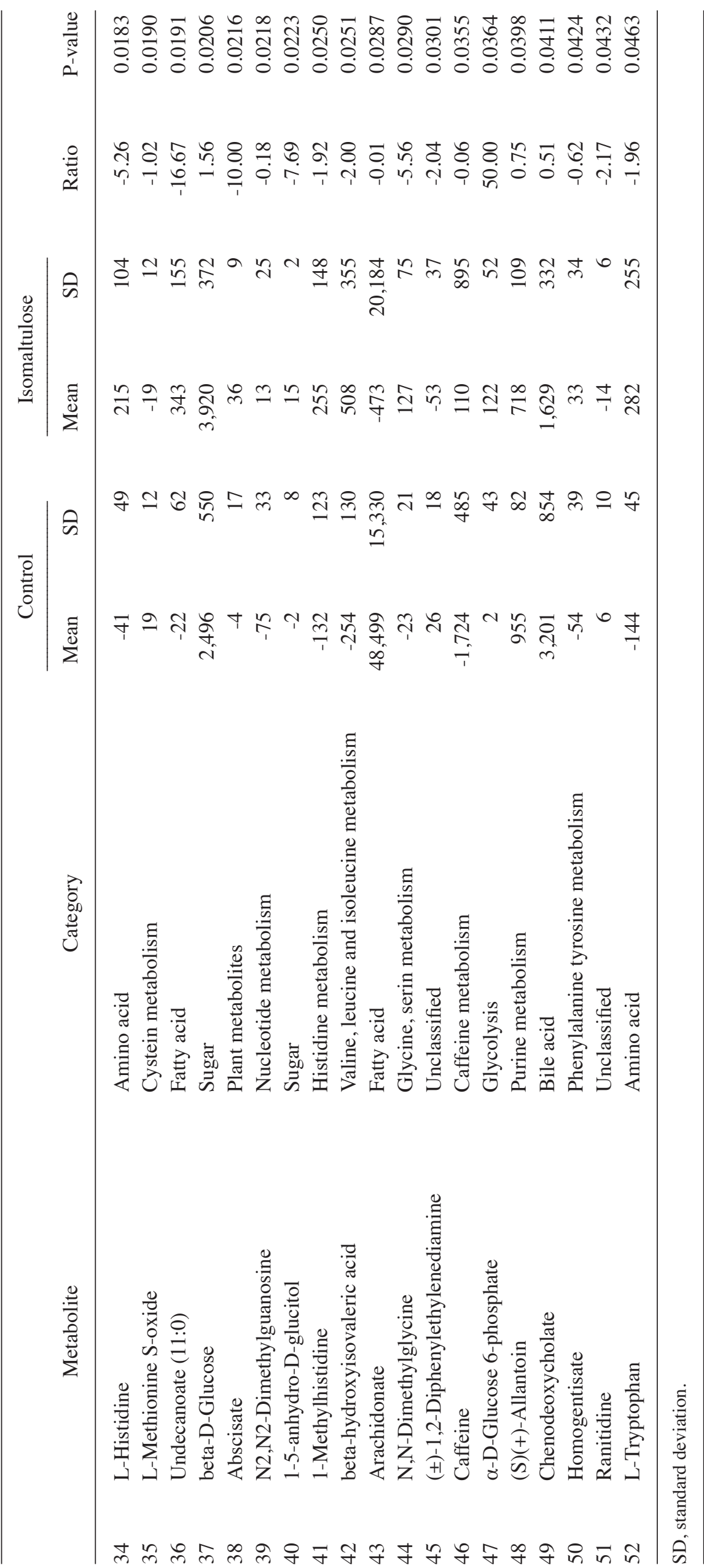


particular, taurodeoxycholic acid, arachidonic acid, and betaine. Thus, isomaltulose may have a beneficial effect on insulin resistance through alterations in various metabolisms, in particular bile acid, fatty acid, and glycine/serine metabolisms.

Isomaltulose has been reported to improve insulin resistance in rats and in patients who are obese $(19,31)$. In agreement with these previous reports, the serum CPR level was significantly decreased in the Isomaltulose group compared to that in the Control group in this study. Thus, we demonstrated that isomaltulose improved insulin resistance in patients with NAFLD. In this study, serum CPR was used for evaluation of insulin resistance instead of immunoreactive insulin (IRI). This was because DCPR is the most significant determinant of insulin secretion increments (30). Moreover, in patients with chronic liver disease, the hepatic insulin degradation rate is decreased, and CPR is more accurate than IRI for the evaluation of insulin secretion (32). Furthermore, the IRI level is affected by hemolysis during blood collection (33) and is inaccurate in the presence of an anti-insulin antibody (34).

The slow digestive rate of isomaltulose is reported to affect secretion of GIP and GLP-1, resulting in a reduction of insulin resistance $(14,15)$. However, in this study, no significant change was seen in GIP or GLP-1 levels. The reason for the discrepancy between previous studies and our study remains unclear, and following are possible explanations (1): The number of enrolled patients may not have had enough power to detect significant changes or (2) since plasma dipeptidyl peptidase-4 activity is accelerated in patients with NAFLD (35), degradation of GIP and GLP-1 could be increased in patients with NAFLD.

To investigate possible mechanisms for isomaltuloseinduced suppression of insulin resistance, we performed a metabolomic analysis and demonstrated that isomaltulose had significant effects on various metabolites in patients with NAFLD. A large number of altered metabolites were categorized into bile acid, fatty acid, or glycine/serine metabolisms. A representative change in each category was an increased taurodeoxycholic acid level, a decreased arachidonic acid level, and an increased betaine level in bile acid, fatty acid, and glycine/serine metabolisms, respectively. The causal relationships between these changes in metabolites and the improvement of insulin resistance remain unclear. However, Qi et al reported that taurodeoxycholic acid may reduce the increase of phospholipids, sphingomyelins, and ceramides induced by a high-fat diet, leading to an improvement of insulin resistance in a mouse model (36). Arachidonic acid is reported to down-regulate the insulin-dependent glucose transporter gene, resulting in an increase in insulin resistance (37). In addition, betaine was recently reported to improve insulin resistance through an activation of forkhead box O1-induced NLRP3 inflammasomes (38). These previous reports support that isomaltulose may have improved insulin resistance potentially through alterations in bile acid, fatty acid, and glycine/serine metabolisms in this study.

The metabolomic analysis also revealed a significant decrease in the serum L-arginine level and a significant increase in the serum L-ornithine level in the isomaltulose

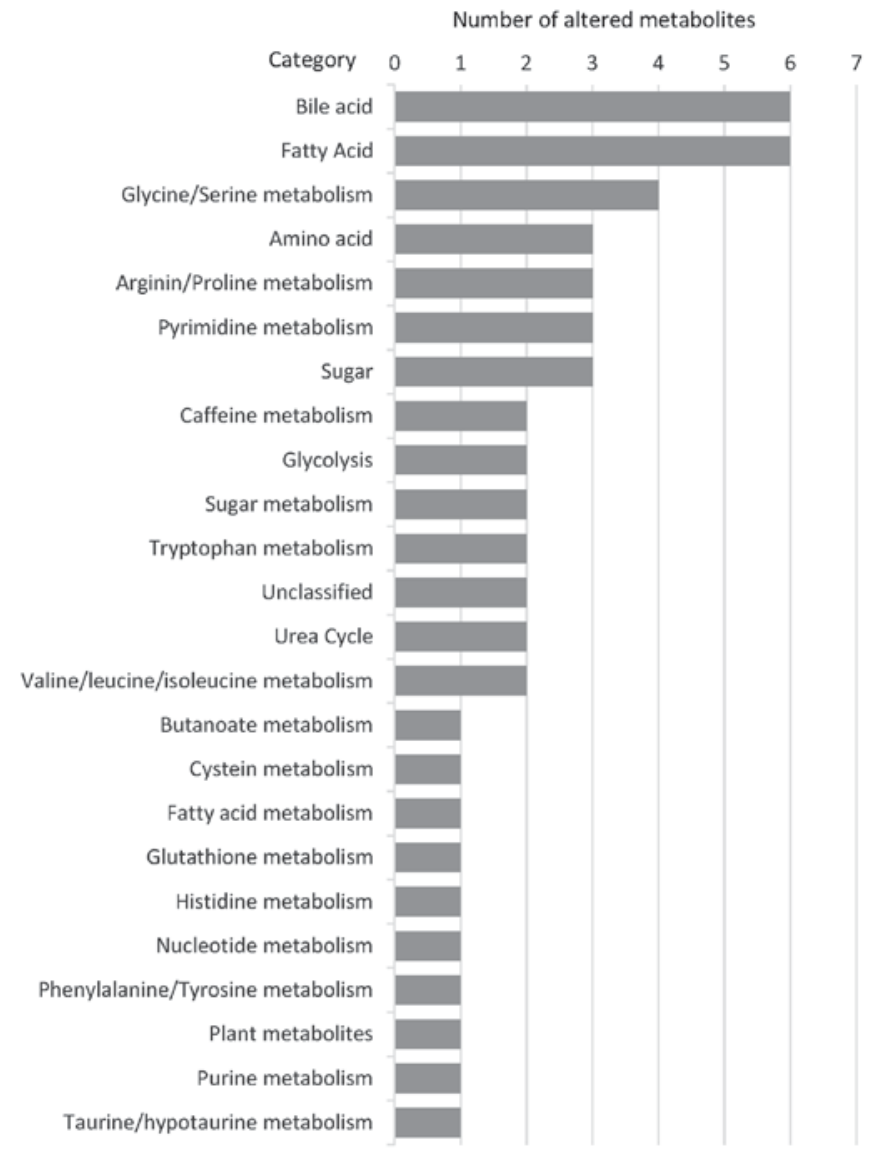

Figure 3. Effect of isomaltulose on serum metabolite levels. With metabolomic analysis, significant alteration was seen in 52 metabolites between the Control and isomaltulose groups. Many of the 52 altered metabolites were seen in categories of bile acid (6 metabolites), fatty acid (6 metabolites), and glycine/serine metabolism (4 metabolites).

group. L-arginine is reported to inhibit gene expression of insulin receptor substrate-1, phosphatidylinositol 3-kinase, and Akt in the insulin signaling pathway, leading to an increase in insulin resistance (39). L-ornithine is reported to regulate growth hormone/insulin-like growth factor-1/insulin-like growth factor-binding protein 3 complex in muscle tissue, leading to improved insulin resistance (40). Taken together, along with alterations in bile acid, fatty acid, and glycine/serine metabolisms, alterations in L-arginine and L-ornithine may contribute to improvement of insulin resistance in patients with NAFLD.

A major limitation of this study is small sample size, and further study is required to verify of our results. In this study, changes in metabolisms were evaluated by a global metabolomic analysis. Since environmental factors including outdoor temperature are known to be associated with insulin resistance and other metabolisms (41-43), isomaltulose or sucrose was administered to the all subjects at the same time on the same day by personal stuffs. Thus, sample size was limited because of the equalization for environmental factors in this study. Another limitation is that taste of isomaltulose is slightly different from that of sucrose and subjects might recognize the difference between isomaltulose and sucrose. However, taste correlates with chemical structure $(44,45)$, and it is impossible to have a control disaccharide with same taste of isomaltulose. 

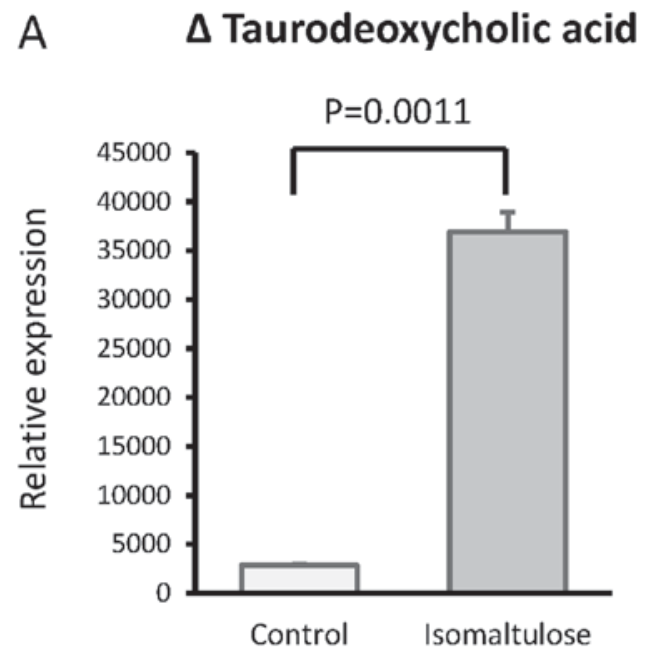

C

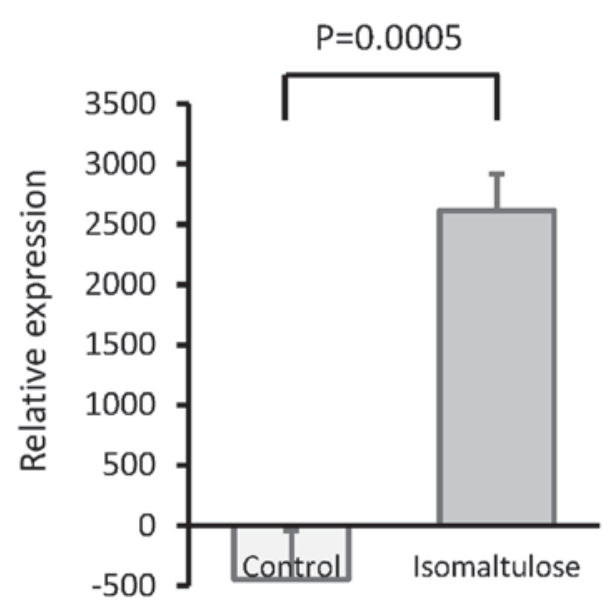

E

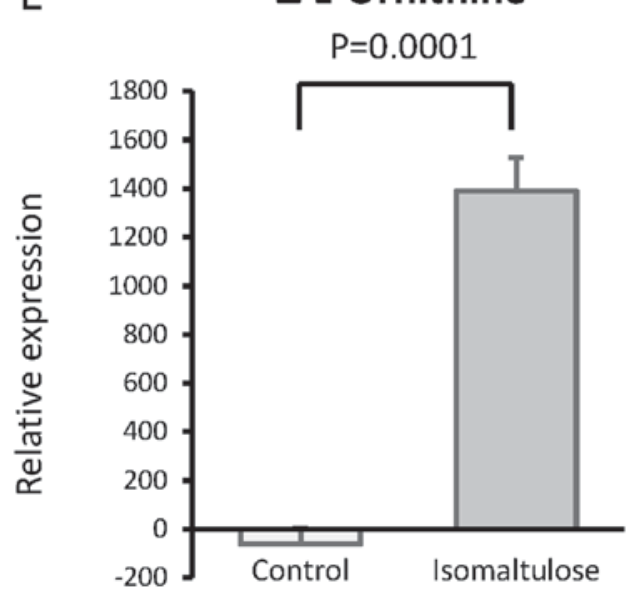

B

$\Delta$ Arachidonic acid

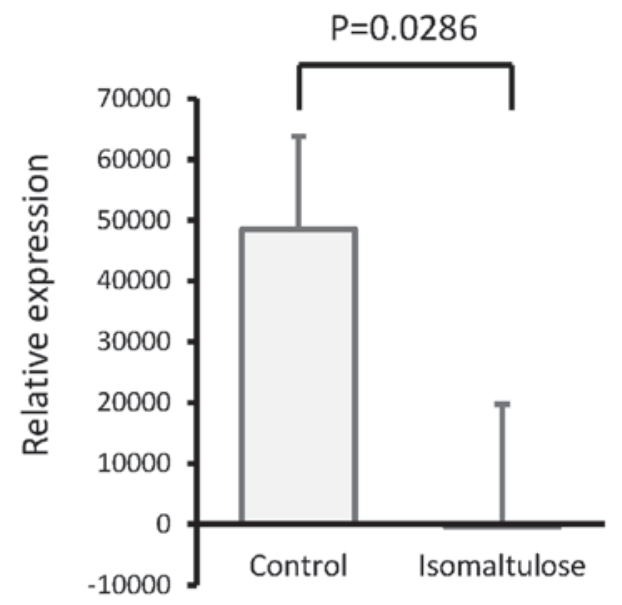

$\Delta$ L-Arginine

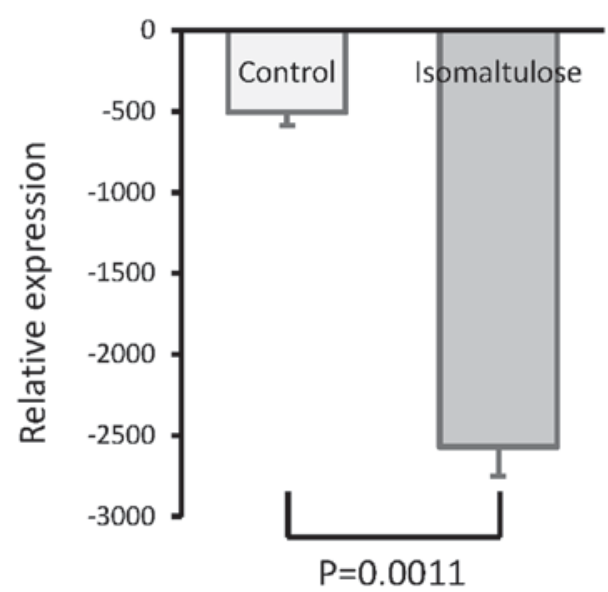

Figure 4. Effect of isomaltulose on serum taurodeoxycholic acid, arachidonic acid, betaine, L-arginine, and L-ornithine levels. The effect of isomaltulose on serum (A) taurodeoxycholic acid, (B) arachidonic acid, (C) betaine, (D) L-arginine, and (E) L-ornithine levels was evaluated by changes in these variables from baseline ( $0 \mathrm{~min})$ to $15 \mathrm{~min}$ after sucrose (Control) or isomaltulose administration. P-values of $<0.05$ were considered significant.

In this study, a random assignment was performed just before the administration and then, subjects were at rest on a bed and fast during the procedure. Thus, the taste difference between isomaltulose and sucrose is considered to have no influence on the results.
In conclusion, we showed that isomaltulose improved insulin resistance in patients with NAFLD. In addition, we revealed that isomaltulose significantly affect various metabolites, including taurodeoxycholic acid, arachidonic acid, and betaine. Thus, isomaltulose may improve insulin resistance 
mainly through alterations in various metabolisms in patients with NAFLD.

\section{Acknowledgements}

Not applicable.

\section{Funding}

No funding was received.

\section{Availability of data and materials}

The datasets analyzed in the current study available from the corresponding author on reasonable request.

\section{Authors' contributions}

TK was involved in the conception and design of the study, acquisition of data, analysis and interpretation of data, drafting of the article, critical revision of the article for important intellectual content, and gave final approval of the version to be submitted. DN and TO were involved in acquisition of data and drafting of the manuscript. TT was involved in acquisition of data, revised the article critically for important intellectual content and gave final approval of the version to be submitted.

\section{Ethics approval and consent to participate}

The study protocol conformed to the ethical guidelines of the 1975 Declaration of Helsinki, as reflected by the prior approval of the institutional review board of The Ethical Committee of Kurume University. All subjects provided written informed consent to participate in this study.

\section{Patient consent for publication}

Not applicable.

\section{Competing interests}

The authors declare that they have no competing interests.

\section{References}

1. Loomba R and Sanyal AJ: The global NAFLD epidemic. Nat Rev Gastroenterol Hepatol 10: 686-690, 2013.

2. Vernon G, Baranova A and Younossi ZM: Systematic review: The epidemiology and natural history of non-alcoholic fatty liver disease and non-alcoholic steatohepatitis in adults. Aliment Pharmacol Ther 34: 274-285, 2011.

3. Kawaguchi T, Izumi N, Charlton MR and Sata M: Branched-chain amino acids as pharmacological nutrients in chronic liver disease. Hepatology 54: 1063-1070, 2011.

4. Kawaguchi T, Yoshida T, Harada M, Hisamoto T, Nagao Y, Ide T, Taniguchi E, Kumemura H, Hanada S, Maeyama M, et al: Hepatitis $\mathrm{C}$ virus down-regulates insulin receptor substrates 1 and 2 through up-regulation of suppressor of cy tokine signaling 3 . Am J Pathol 165: 1499-1508, 2004.

5. Younossi Z and Henry L: Contribution of alcoholic and nonalcoholic fatty liver disease to the burden of liver-related morbidity and mortality. Gastroenterology 150: 1778-1785, 2016.

6. Belfort R, Harrison SA, Brown K, Darland C, Finch J, Hardies J, Balas B, Gastaldelli A, Tio F, Pulcini J,et al: A placebo-controlled trial of pioglitazone in subjects with nonalcoholic steatohepatitis. N Engl J Med 355: 2297-2307, 2006.
7. Armstrong MJ, Gaunt P, Aithal GP, Barton D, Hull D, Parker R, Hazlehurst JM, Guo K; LEAN trial team, Abouda G, et al: Liraglutide safety and efficacy in patients with non-alcoholic steatohepatitis (LEAN): A multicentre, double-blind,randomised, placebo-controlled phase 2 study. Lancet 387: 679-690, 2016.

8. Akuta N, Watanabe C,Kawamura Y,Arase Y,Saitoh S, Fujiyama S, Sezaki H, Hosaka T, Kobayashi M, Kobayashi M, et al: Effects of a sodium-glucose cotransporter 2 inhibitor in nonalcoholic fatty liver disease complicated by diabetes mellitus: Preliminary prospective study based on serial liver biopsies. Hepatol Commun 1: 46-52, 2017.

9. Lina BA, Jonker D and Kozianowski G: Isomaltulose (Palatinose): A review of biological and toxicological studies. Food Chem Toxicol 40: 1375-1381, 2002.

10. Dahlqvist A, Auricchio S, Semenza G and Prader A: Human intestinal disaccharidases and hereditary disaccharide intolerance. The hydrolysis of sucrose, isomaltose, palatinose (isomaltulose), and a 1,6-alpha-oligosaccharide (isomalto-oligosaccharide) preparation. J Clin Invest 42: 556-562, 1963.

11. Okuno M, Kim MK, Mizu M, Mori M, Mori H and Yamori Y: Palatinose-blended sugar compared with sucrose: Different effects on insulin sensitivity after 12 weeks supplementation in sedentary adults. Int J Food Sci Nutr 61: 643-651, 2010.

12. Mu W, Li W, Wang X, Zhang T and Jiang B: Current studies on sucrose isomerase and biological isomaltulose production using sucrose isomerase. Appl Microbiol Biotechnol 98: 6569-6582, 2014.

13. Tonouchi H, Yamaji T, Uchida M, Koganei M, Sasayama A, Kaneko T, Urita Y, Okuno M, Suzuki K, Kashimura J and Sasaki H: Studies on absorption and metabolism of palatinose (isomaltulose) in rats. Br J Nutr 105: 10-14, 2011.

14. Keyhani-Nejad F, Irmler M, Isken F, Wirth EK, Beckers J, Birkenfeld AL and Pfeiffer AF: Nutritional strategy to prevent fatty liver and insulin resistance independent of obesity by reducing glucose-dependent insulinotropic polypeptide responses in mice. Diabetologia 58: 374-383, 2015.

15. Ang M and Linn T: Comparison of the effects of slowly and rapidly absorbed carbohydrates on postprandial glucose metabolism in type 2 diabetes mellitus patients: A randomized trial. Am J Clin Nutr 100: 1059-1068, 2014.

16. Maresch CC, Petry SF, Theis S, Bosy-Westphal A and Linn T: Low glycemic index prototype isomaltulose-update of clinical trials. Nutrients 9: pii: E381, 2017.

17. Suzuki M, Shido D, Goto K, Ohno Y, Miyasaka K and Mizu M: Effects of a palatinose-containing diet with exercise on progression of diabetic nephropathy and metabolic syndrome in obese-diabetic rats. Euro J Sports Exerc Sci 5: 27-36, 2017.

18. Arai H, Mizuno A, Sakuma M, Fukaya M, Matsuo K, Muto K, Sasaki H, Matsuura M, Okumura H, Yamamoto H, et al: Effects of a palatinose-based liquid diet (Inslow) on glycemic control and the second-meal effect in healthy men. Metabolism 56: 115-121, 2007.

19. König D, Theis S, Kozianowski G and Berg A: Postprandial substrate use in overweight subjects with the metabolic syndrome after isomaltulose (Palatinose ${ }^{\mathrm{TM}}$ ) ingestion. Nutrition 28: 651-656, 2012.

20. Yamori Y, Mori M, Hori H, Kashimura J, Sakuma T, Ishikawa PM, Moriguchi E and Moriguchi Y: Japanese perspective on reduction in lifestyle disease risk in immigrant Japanese Brazilians: A double-blind, placebo-controlled intervetion study on palatinose. Clin Exp Pharmacol Physiol 34: S5-S7, 2007.

21. Postic C and Girard J: Contribution of de novo fatty acid synthesis to hepatic steatosis and insulin resistance: Lessons from genetically engineered mice. J Clin Invest 118: 829-838, 2008.

22. Legry V, Francque S, Haas JT, Verrijken A, Caron S, Chávez-Talavera O, Vallez E, Vonghia L, Dirinck E, Verhaegen A, et al: Bile acid alterations are associated with insulin resistance, but not with NASH, in obese subjects. J Clin Endocrinol Metab 102: 3783-3794, 2017.

23. Weckwerth $\mathrm{W}$ and Fiehn O: Can we discover novel pathways using metabolomic analysis? Curr Opin Biotechnol 13: 156-160, 2002.

24. Li J, Liu Z, Guo M, Xu K, Jiang M, Lu A and Gao X: Metabolomics profiling to investigate the pharmacologic mechanisms of berberine for the treatment of high-fat diet-induced nonalcoholic steatohepatitis. Evid Based Complement Alternat Med 2015: 897914, 2015.

25. Watanabe S, Hashimoto $\mathrm{E}$, Ikejima $\mathrm{K}$, Uto $\mathrm{H}$, Ono $\mathrm{M}$, Sumida Y, Seike M, Takei Y, Takehara T, Tokushige K, et al: Evidence-based clinical practice guidelines for nonalcoholic fatty liver disease/nonalcoholic steatohepatitis. Hepatol Res 45: 363-377, 2015. 
26. Kawaguchi T, Ide T, Taniguchi E, Hirano E, Itou M, Sumie S, Nagao Y, Yanagimoto C, Hanada S, Koga $\mathrm{H}$ and Sata M: Clearance of $\mathrm{HCV}$ improves insulin resistance, beta-cell function, and hepatic expression of insulin receptor substrate 1 and 2 . Am J Gastroenterol 102: 570-576, 2007.

27. Kawaguchi $\mathrm{T}$, Kuromatsu R, Ide T, Taniguchi E, Itou M, Sakata M, Abe M, Sumie S and Sata M: Thrombocytopenia, an important interfering factor of antiviral therapy and hepatocellular carcinoma treatment for chronic liver diseases. Kurume Med J 56: 9-15, 2009.

28. Itou M, Kawaguchi T, Taniguchi E, Sumie S, Oriishi T, Mitsuyama K, Tsuruta O, Ueno T and Sata M: Altered expression of glucagon-like peptide-1 and dipeptidyl peptidase IV in patients with HCV-related glucose intolerance. J Gastroenterol Hepatol 23: 244-251, 2008

29. Tanabe K, Kitagawa K, Kojima N and Iijima S: Multifucosylated alpha-1-acid glycoprotein as a novel marker for hepatocellular carcinoma. J Proteome Res 15: 2935-2944, 2016.

30. Yoshikawa A, Kozawa J, Okita K, Yoneda S, Okauchi Y, Uno S, Iwahashi H, Ohira T, Takiuchi D, Eguchi H, et al: Preoperative insulin secretion ability and pancreatic parenchymal thickness as useful parameters for predicting postoperative insulin secretion in patients undergoing pancreaticoduodenectomy. Endocr J 59: 383-392, 2012

31. Arai H, Mizuno A, Matsuo K, Fukaya M, Sasaki H, Arima H, Matsuura M, Taketani Y, Doi T and Takeda E: Effect of a novel palatinose-based liquid balanced formula (MHN-01) on glucose and lipid metabolism in male Sprague-Dawley rats after short- and long-term ingestion. Metabolism 53: 977-983, 2004.

32. Iwasaki Y, Ohkubo A, Kajinuma H, Akanuma Y and Kosaka K: Degradation and secretion of insulin in hepatic cirrhosis. J Clin Endocrinol Metab 47: 774-779, 1978.

33. Matulevicius V, Coculescu $M$ and Urbanavicius V: Immunoreactive insulin in haemolyzed erythrocytes of normal humans. Physiologie 17: 217-219, 1980.

34. Sakata S, Nagai K, Imai T, Komaki T and Miura K: A case of diabetes mellitus associated with anti-insulin autoantibodies without previous insulin injection. J Endocrinol Invest 10: 407-411, 1987

35. Zheng T, Chen B, Yang L, Hu X, Zhang X, Liu H and Qin L: Association of plasma dipeptidyl peptidase-4 activity with non-alcoholic fatty liver disease in nondiabetic Chinese population. Metabolism 73: 125-134, 2017.
36. Qi Y, Jiang C, Cheng J, Krausz KW, Li T, Ferrell JM, Gonzalez FJ and Chiang JY: Bile acid signaling in lipid metabolism: Metabolomic and lipidomic analysis of lipid and bile acid markers linked to anti-obesity and anti-diabetes in mice. Biochim Biophys Acta 1851: 19-29, 2015.

37. Tebbey PW, McGowan KM, Stephens JM, Buttke TM and Pekala PH: Arachidonic acid down-regulates the insulin-dependent glucose transporter gene (GLUT4) in 3T3-L1 adipocytes by inhibiting transcription and enhancing mRNA turnover. J Biol Chem 269: 639-644, 1994.

38. Kim DH, Kim SM, Lee B, Lee EK, Chung KW, Moon KM, An HJ, Kim KM, Yu BP and Chung HY: Effect of betaine on hepatic insulin resistance through FOXO1-induced NLRP3 inflammasome. J Nutr Biochem 45: 104-114, 2017.

39. Liang H, Habte-Tsion HM, Ge X, Ren M, Xie J, Miao L, Zhou Q, Lin Y and Pan W: Dietary arginine affects the insulin signaling pathway, glucose metabolism and lipogenesis in juvenile blunt snout bream Megalobrama amblycephala. Sci Rep 7: 7864, 2017.

40. Zajac A, Poprzecki S, Zebrowska A, Chalimoniuk M and Langfort J: Arginine and ornithine supplementation increases growth hormone and insulin-like growth factor-1 serum levels after heavy-resistance exercise in strength-trained athletes. J Strength Cond Res 24: 1082-1090, 2010

41. Betz MJ, Slawik M, Lidell ME, Osswald A, Heglind M, Nilsson D, Lichtenauer UD, Mauracher B, Mussack T, Beuschlein F and Enerbäck S: Presence of brown adipocytes in retroperitoneal fat from patients with benign adrenal tumors: Relationship with outdoor temperature. J Clin Endocrinol Metab 98: 4097-4104, 2013.

42. Wei Q, Lee JH, Wang H, Bongmba OYN, Wu CS, Pradhan G, Sun Z, Chew L, Bajaj M, Chan L, et al: Adiponectin is required for maintaining normal body temperature in a cold environment. BMC Physiol 17: 8, 2017.

43. Davies JW and Liljedahl SO: The effect of environmental temperature on the metabolism and nutrition of burned patients. Proc Nutr Soc 30: 165-172, 1971.

44. Hamor GH: Correlation of chemical structure and taste in the saccharin series. Science 134: 1416-1417, 1961.

45. Dagan-Wiener A, Nissim I, Ben Abu N, Borgonovo G, Bassoli A and Niv MY: Bitter or not? BitterPredict, a tool for predicting taste from chemical structure. Sci Rep 7: 12074, 2017.

This work is licensed under a Creative Commons Attribution-NonCommercial-NoDerivatives 4.0 International (CC BY-NC-ND 4.0) License. 